Article

\title{
Investigation of the Influence of Neutron Irradiation on Cladded Nuclear Reactor Pressure Vessel Steel Blocks by Magnetic Adaptive Testing
}

\author{
Gábor Vértesy ${ }^{1, * \mathbb{D}}$, Antal Gasparics ${ }^{1}\left(\mathbb{D}\right.$, Ildikó Szenthe ${ }^{1}$ and Inge Uytdenhouwen ${ }^{2}(\mathbb{D}$ \\ 1 Centre for Energy Research, Institute of Technical Physics and Materials Science, 1121 Budapest, Hungary; \\ gasparics.antal@ek-cer.hu (A.G.); szenthe.ildiko@ek-cer.hu (I.S.) \\ 2 SCK CEN Belgian Nuclear Research Centre, 2400 Mol, Belgium; inge.uytdenhouwen@sckcen.be \\ * Correspondence: vertesy.gabor@ek-cer.hu
}

check for

updates

Citation: Vértesy, G.; Gasparics, A.;

Szenthe, I.; Uytdenhouwen, I.

Investigation of the Influence of

Neutron Irradiation on Cladded

Nuclear Reactor Pressure Vessel Steel

Blocks by Magnetic Adaptive Testing.

Appl. Sci. 2022, 12, 2074. https://

doi.org/10.3390/app12042074

Academic Editor: Giuseppe

Lacidogna

Received: 26 January 2022

Accepted: 15 February 2022

Published: 16 February 2022

Publisher's Note: MDPI stays neutral with regard to jurisdictional claims in published maps and institutional affiliations.

Copyright: (C) 2022 by the authors. Licensee MDPI, Basel, Switzerland. This article is an open access article distributed under the terms and conditions of the Creative Commons Attribution (CC BY) license (https:// creativecommons.org/licenses/by/ $4.0 /)$.

\begin{abstract}
The influence of neutron irradiation degradation on reactor pressure vessel steel was investigated. Large blocks were irradiated by neutrons in the BR2 reactor at a low irradiation temperature. They were measured by a nondestructive magnetic method, magnetic adaptive testing, before and after the neutron irradiation. It was shown that the modification of the magnetic parameters due to the neutron irradiation was well detectable by this nondestructive method. It was also shown that the influence of neutron irradiation could be detected with a reasonable signal-to-noise ratio through the cladding. The present results are considered as a first step towards the application of magnetic adaptive testing in the nuclear industry.
\end{abstract}

Keywords: neutron irradiation; reactor pressure vessel steel; magnetic nondestructive evaluation; magnetic adaptive testing

\section{Introduction}

In many countries, nuclear power plants play a deterministic role in energy production. Their safety aspects are very important-safe operation must be guaranteed at any time. In case of light water (pressurized and boiling) reactors, the reactor pressure vessel (RPV) is the most important, not replaceable component. During the whole lifetime of a nuclear power plant, the material of RPV is under continuous neutron radiation. The fast neutrons to material structure interaction processes generate modification of the mechanical properties (decrease of toughness, increase of yield stress, hardness, and tensile strength) [1]. The most important ageing phenomenon is the neutron irradiation generated material embrittlement. Radiation embrittlement is indirectly estimated from surveillance programs monitoring the reactor vessel degradation. Surveillance capsules contain specimens from relevant materials, including base metals and welds representative of the RPV. By applying the well-known Charpy impact method, the ductile to brittle transition temperature (DBTT) can be determined [2]. Different types of nondestructive methods were suggested for monitoring the material degradation. In contrast to destructive tests, nondestructive tests do not directly measure the mechanical properties of the investigated material. Before practical application of each nondestructive test, the results must be rigorously correlated to the applied destructive ones in many series of investigations.

Considering that RPV steel is ferromagnetic, magnetic methods seem to be useful nondestructive measurement techniques for measuring material degradation. The reason is that a well-known correlation that has been proven many times exists between magnetic behaviour and macroscopic physical properties of the materials. The physical principle is detailed in [3] while reference [4] contains a general overview about the practical application of different nondestructive magnetic techniques. 
One of the non-destructive techniques is the magnetic adaptive testing (MAT) $[5,6]$. This is a special type of measurement of magnetic hysteresis loops. The main advantages of this method are the following: In contrast to the traditional hysteresis measurement, it is not the major loop and its few key parameters that are measured, but a series of minor ones. This way, a big data pool is generated and those descriptors are chosen from this data pool, which characterize the best degradation of the investigated material. As is shown in [6], this method offers parameters that characterize the correlation between magnetic descriptors and independent variables (embrittlement, ageing, fatigue, the degree of thermal treatment, plastic and elastic deformation, neutron irradiation, etc.) more sensitively and more reliably than traditional magnetic parameters. In addition, there is no need for magnetic saturation of the investigated samples, which can be hardly carried out in practical applications. Evidently, this method also has some drawbacks, which are as follows: The method does not provide absolute magnetic quantities, as it is suitable only for comparative measurement. However, if the method is "taught" on a series of reference samples, a calibration curve can be generated. Thus, parameters of any unknown sample from a similar series of samples can be characterized by one single measurement, if the parameters of the measurement is kept fixed. Another drawback of the method is (similarly to any other magnetic measurement, where a magnetizing yoke is placed on the samples' surface) that the surface condition has a great influence on the detected magnetic signal. This problem can also be handled somehow by using a non-magnetic spacer between the sample and the yoke, as described in detail in [6].

Recently, several papers were published, where neutron embrittlement of RPV steels was investigated by MAT [7-9] on Charpy specimens. DBTT values were compared with MAT parameters on RPV steels, which were irradiated by neutrons.

In this work, instead of measurement of Charpy specimens, the MAT is applied on neutron irradiated RPV steel blocks in order to simulate the direct measurement on the reactor vessel. Measurements were performed on two sides of the blocks-one from the base material side and one on the cladded sides of the blocks.

\section{Materials and Methods}

\subsection{Sample Preparation}

In order to characterize damage caused by neutron irradiation, a Western RPV material A508 Cl.2 [10,11] was used. A large part of the Lemoniz reactor vessel, a Spanish reactor of Western type that was not operational, was chosen to manufacture block specimens at SCK CEN. The samples have a mixed tempered ferrite-bainite microstructure. The chemical composition of the material is given in Table 1.

Table 1. Chemical composition (wt.\%) of the A508 Cl.2 base metal as measured by optical emission spectroscopy at SCK CEN on a Charpy specimen.

\begin{tabular}{ccccccccc}
\hline $\mathbf{C}$ & $\mathbf{M n}$ & $\mathbf{S i}$ & $\mathbf{S}$ & $\mathbf{P}$ & $\mathbf{C r}$ & $\mathbf{N i}$ & $\mathbf{M o}$ & $\mathbf{C u}$ \\
\hline 0.201 & 0.578 & 0.27 & 0.0085 & 0.0091 & 0.372 & 0.668 & 0.599 & 0.0472 \\
\hline
\end{tabular}

Large un-cladded (block type III) and cladded (block type IV) base material blocks with fixed dimensions were used. The geometry of the samples are given in Figure 1, while the whole set of investigated specimens can be seen in Figure 2. Cladding is clearly seen on the blocks of the left side of Figure 2.

The blocks were irradiated in the BR2 reactor of the Belgian Nuclear Research Centre to various neutron fluence levels at low irradiation temperature $\left(<\sim 100{ }^{\circ} \mathrm{C}\right)$ [12]. The low irradiation temperature was chosen to induce as much neutron irradiation damage as possible but also to reduce the thermal gradient that is induced by $\gamma$-heating.

Half of the blocks had a uniform neutron fluence profile and half were irradiated with an attenuation fluence profile. Due to the attenuation profile in 6 of the blocks, more 
than 12 fluence conditions were available. The range of the fast neutron fluence of the A508 Cl.2 blocks is between 1.48 and $21.40 \times 10^{19} \mathrm{n} / \mathrm{cm}(\mathrm{E}>1 \mathrm{MeV})$ [12].
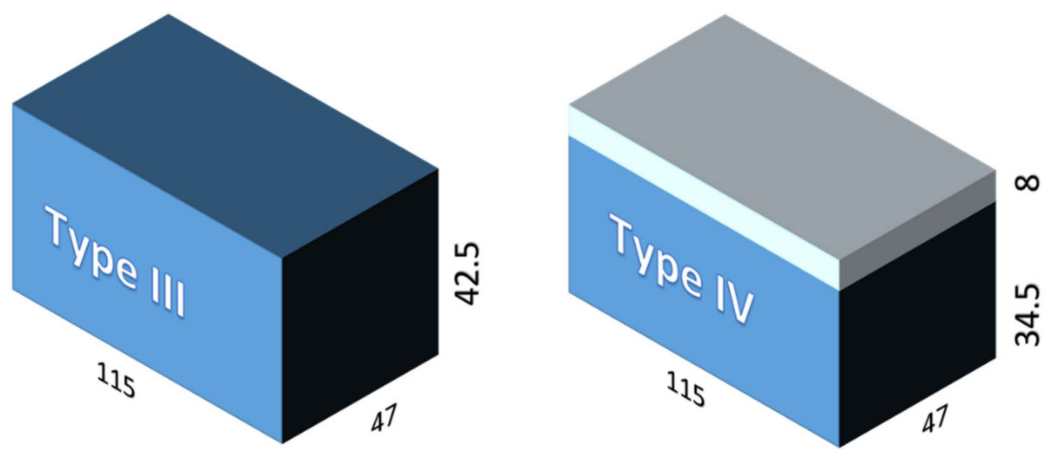

Figure 1. Geometry and type of the measured blocks without and with cladding [12].

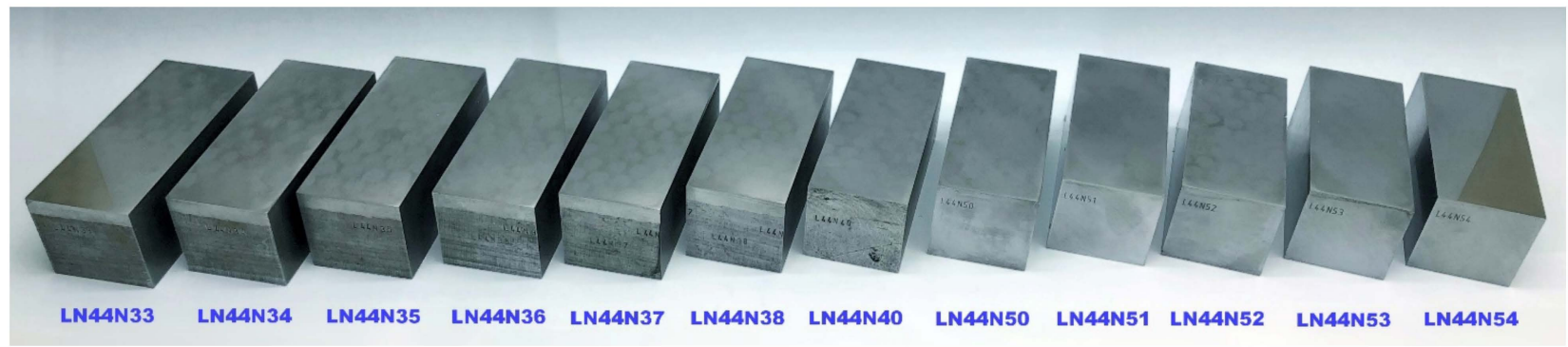

Figure 2. Photograph and IDs of all investigated blocks [12].

\subsection{Magnetic Adaptive Testing}

The magnetic adaptive testing (MAT) technique measures systematically the series of minor magnetic permeability loops. During the measurement, the amplitude of the magnetizing current is increased step-by-step, starting from the demagnetized state. The magnetizing current has a triangular waveform. The time-dependent increase of the magnetizing field is linear. It means that the voltage, which is generated in the pick-up coil, is proportional to the differential permeability of the measured specimen. Series of the detected permeability loops, measured on different blocks, can be seen in Figure 3. In this figure, all minor loops of all measured samples are shown. The figure is a bit overcrowded, but the main purpose is to illustrate the draw measured data in one graph. The points of these loops are the input for further data evaluation. Each point represents a kind of information about the specimen's magnetic behaviour.

It is also seen in this figure, especially when the magnified part is considered, that there are significant differences in the magnetic properties of the different samples. This is the reason for the large scatter of reference samples (having zero neutron level) when the MAT descriptor as a function of the irradiated neutron fluence is considered in the Result and Discussion section. The phenomenon is surprising and still not understood, considering that the samples were cut from the same large block. A very similar scatter of magnetic parameters of not-irradiated Charpy geometry samples were observed very recently [9]. A systematic and careful further analysis of this phenomenon is necessary. Such research will be conducted, but is currently out of scope in this work.

A magnetizing yoke (a half transformer core, made of laminated Fe-Si sheets) put on the top of the sample is used for magnetizing the sample. The exciting coil and the pick-up coil are wound on the yoke legs. The cross-section of the leg of the yoke was $19 \times 16 \mathrm{~mm}^{2}$ in our experiments. The probe was designed for usage in a hot cell. The replacement of the samples can be done easily by a manipulator. An example of measurement of the cladded RPV steel block is shown in Figure 4. The magnetizing yoke is put on the top of the block (on the cladding side). 


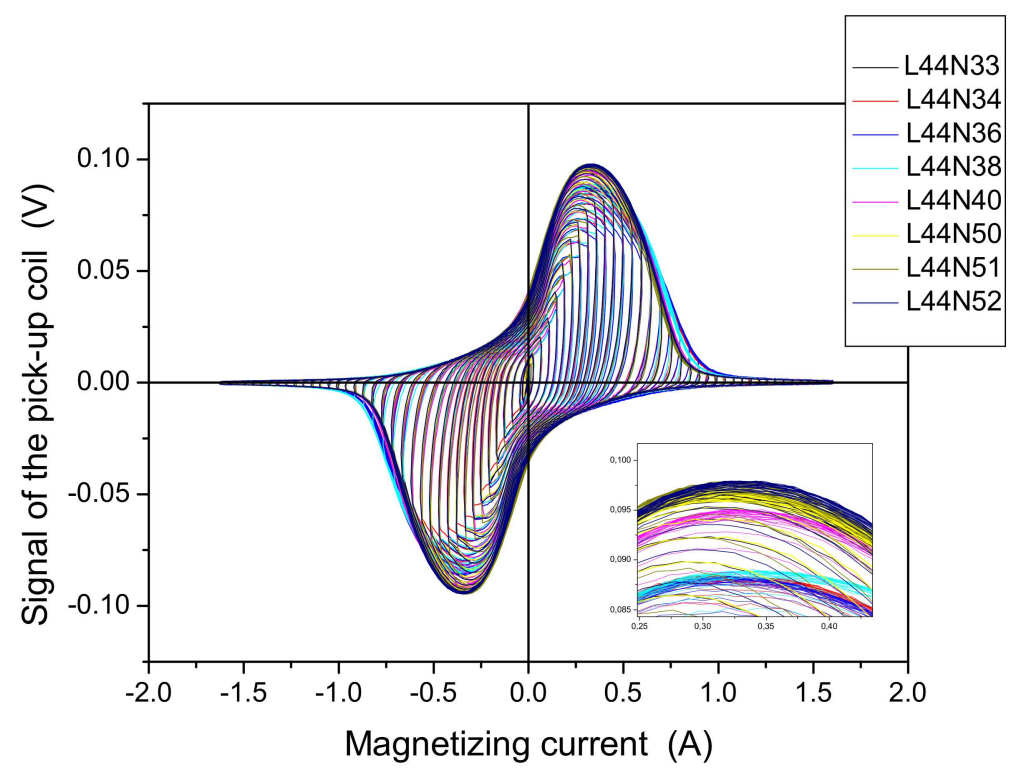

Figure 3. The measured permeability loops of the investigated, not-irradiated samples, measured on the bottom side. Input: magnified part of the loops.

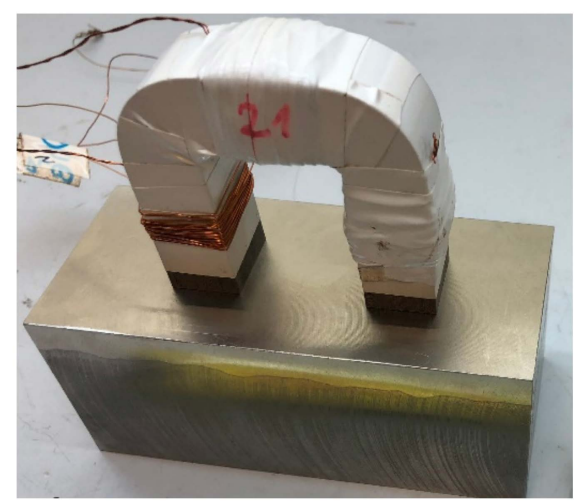

Figure 4. The magnetizing yoke, placed on the top of a cladded RPV steel block.

The probe itself (without sample), designed for hot cell measurements, can be seen in the left side of Figure 5, while the probe with the sample on the top, inside the hot cell, is shown in the right side of Figure 5.

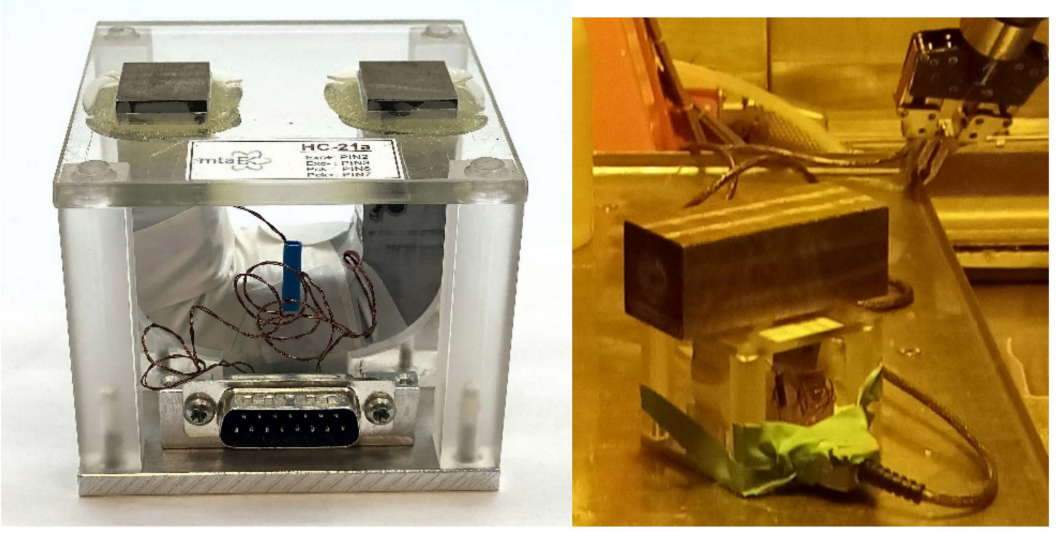

Figure 5. Probe, designed for hot cell MAT measurements (left), and the same probe inside the hot cell, with the sample to be measured (right). 
As presented in Figure 3, regular loops with large amplitudes are experienced when the highly ferromagnetic base material is measured. The situation is drastically different on the top side, if it is covered by cladding. Cladding is an austenitic, highly non-magnetic material. This layer means a large, practically not magnetic gap between the magnetizing yoke and the ferromagnetic part of the sample. As a consequence, a significant part of the exciting magnetic flux closes outside of the ferromagnetic sample; only a very small part of this flux can be pumped into the material. In this case, a very weak signal is experienced, as shown in Figure 6 (note that the vertical scale is different from Figure 3). Nevertheless, a kind of permeability loop with a detectable maximum can be measured, even in this case.

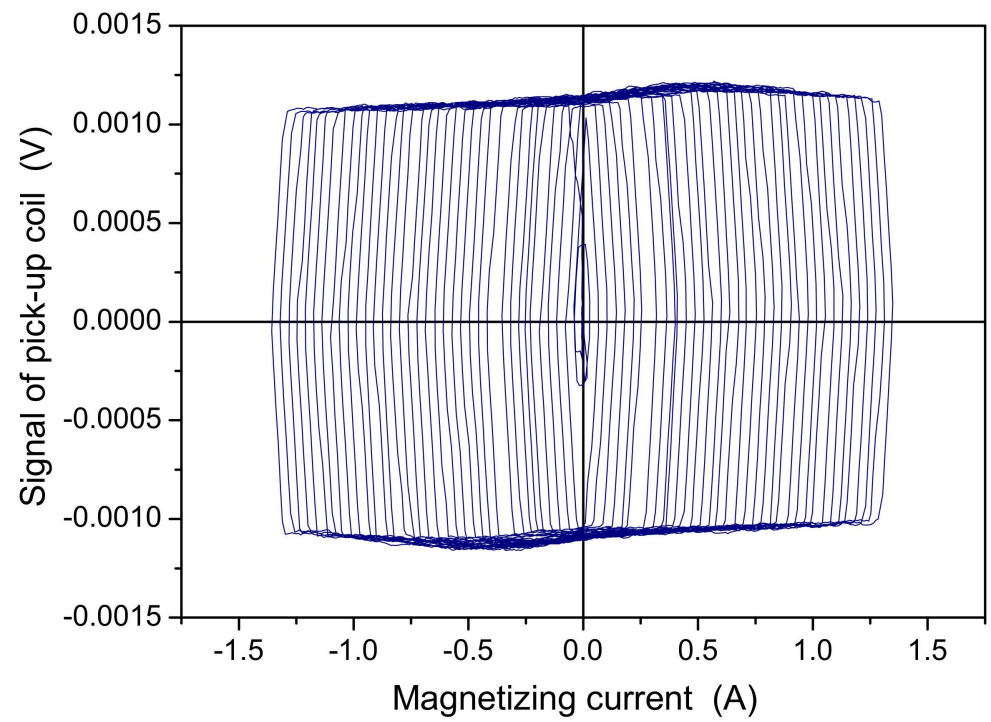

Figure 6. The measured permeability loops of not-irradiated sample L44N33, measured on the cladded (top) side.

The second step of MAT technique is the evaluation of the measured permeability loops. Matrices are calculated from raw data. Primary matrix is the $\mu$ permeability matrix, which is directly calculated from the measured permeability loops. However, in some cases, other matrices can offer more information about the degradation of the material. $D a$-matrix is a matrix of the derivative of permeability with respect to the magnetizing field (actually the first derivative of permeability with respect to the field, or second derivative of the magnetic induction with respect to the field). Ia-matrix is a matrix of the integrated permeability along the field (a hysteresis loops matrix).

Thus, a large data pool is obtained, and these matrix elements magnetically characterize the measured samples. All matrix elements are compared with the same matrix elements obtained on the reference sample. These descriptors are considered to be functions of independent parameters that describe the modification of the material caused by any external influence. The goal of the MAT evaluation is to determine those degradation functions, which characterize the best actual modification of the material under a certain external influence. In this work, this external influence is the neutron fluence.

The final parameter after a procedure of MAT measurement and evaluation, chosen as the best, is called "Optimally chosen MAT descriptor". This is the parameter (characterized by an $h_{a}$ magnetizing field value and an $h_{b}$ minor loop amplitude value) chosen from the above-mentioned large data pool, showing the best correlation with the independent parameter. This descriptor ensures the highest sensitivity and has good reproducibility at the same time. The complete MAT procedure and evaluation is detailed in [6]. It should be realized that the "Optimally chosen MAT descriptor" chooses only a certain amount of data from the total data available.

The error of MAT descriptors was carefully analyzed in [13]. It was demonstrated that the error of the "Optimally chosen MAT descriptor" is not more than $1 \%$. This error 
has three sources: One is the electronic error of measurement, which is almost negligible. The second is the error of repeated measurements due to removing and putting back the measuring head on the sample, or repeating the measurements on different days. This error is larger, but still not that significant. The third one is related to the whole MAT evaluation (calculating matrices) process when repeating different measurements. The final conclusion of this error analysis is that the frequently observed very large scatter of measurements, performed on specimens taken under the same conditions, cannot be explained by the experimental error of magnetic measurements.

\section{Results and Discussion}

Magnetic measurements were performed on the same block samples before and after neutron irradiation, on steel and cladded sides, respectively.

In Figure 7, the "Optimally chosen MAT descriptor" is plotted as a function of neutron fluence. In this case, this descriptor is an element of $D a$ matrix, characterized by $h_{a}=$ $-600 \mathrm{~mA}$ and $h_{b}=1200 \mathrm{~mA}$. During evaluation, the Da matrix elements of irradiated samples were normalized by the corresponding $D a$ matrix element of one of the reference (not irradiated, L44N36) samples. This is the meaning of "Optimally chosen normalized MAT descriptor". As is seen, the descriptors of reference samples are around 1, as they should be, but they scatter a lot. This means that the investigated samples behave differently from a magnetic point of view, even before neutron irradiation. Measurements were performed on the base material, both on top and bottom sides of the samples without cladding. After neutron irradiation, the top and bottom side received a different neutron fluence.

Similar to the results reported previously on Charpy specimens [8], neutron irradiation caused measurable changes in the material properties. Neutron irradiation at low fluences generates a significant modification of the material properties [14], while a further increase of the neutron fluence causes a saturation for higher fluence levels. This means that the present results on RPV blocks are in good correlation with the expectation, based on previous measurement. Larger $\left(15-25 \times 10^{19} \mathrm{n} / \mathrm{cm}^{2}\right)$ neutron fluence caused about $30-40 \%$ modification of the "Optimally chosen MAT descriptors" of the investigated material.

However, it is also seen that the measured points scatter a lot (see Figure 7), much more than what can be explained by the measurement error of the magnetic measurements. This fact needs further analysis, which is out of the scope of this work, but will be done in our future work. Nevertheless, considering that magnetically the blocks were rather different even before irradiation, the scatter of points after irradiation is not so surprising any more.

As mentioned previously, during neutron irradiation, some blocks had a uniform neutron fluence profile and some others were irradiated with an attenuation fluence profile. Since the studied MAT setup has a depth sensitivity profile, it is possible to consider the above presented results from this point of view, i.e., to see if this difference of irradiation caused any difference in the fluence dependent magnetic parameters. The results are shown in Figure 8. This figure contains the same points as Figure 7, but the two sets of samples are indicated by different symbols. Furthermore, for all the reference (not irradiated) samples, the magnetic parameter was taken as exactly equal to 1 .

It is seen that different irradiation conditions did not cause significant change in the magnetic descriptor vs. neutron fluence correlation.

Magnetic measurements were also performed on the cladding side and included in Figures 7 and 8. In spite of the weak magnetic signal (see Figure 6), MAT evaluation resulted in a reasonable correlation between the magnetic parameters and the estimated neutron fluence. In other words, the influence of the neutron irradiation can be followed with acceptable signal-to-noise ratio by this technique. The tendency of the material degradation was found to be very similar as in the case of the hardness measurements performed directly on the base material. In case of MAT evaluation of cladding side measurements, the optimal MAT descriptor is an element of the reciprocal I $a$ matrix, and is characterized 
by $h_{a}=150 \mathrm{~mA}$ and $h_{b}=1000 \mathrm{~mA}$ field values. The results are shown in Figure 9 . The sensitivity of the measurement is lower than when the measurements were performed on base material without cladding: $10-12 \%$ modification of magnetic behaviour of the investigated material was found. The scatter of points is relatively low, much lower than in the case when base material was directly measured. This fact is a direct confirmation with our earlier results that by applying a nonmagnetic spacer (artificial air gap), the scatter of points can be reduced [15]. In this case, the cladding acts as a nonmagnetic spacer.

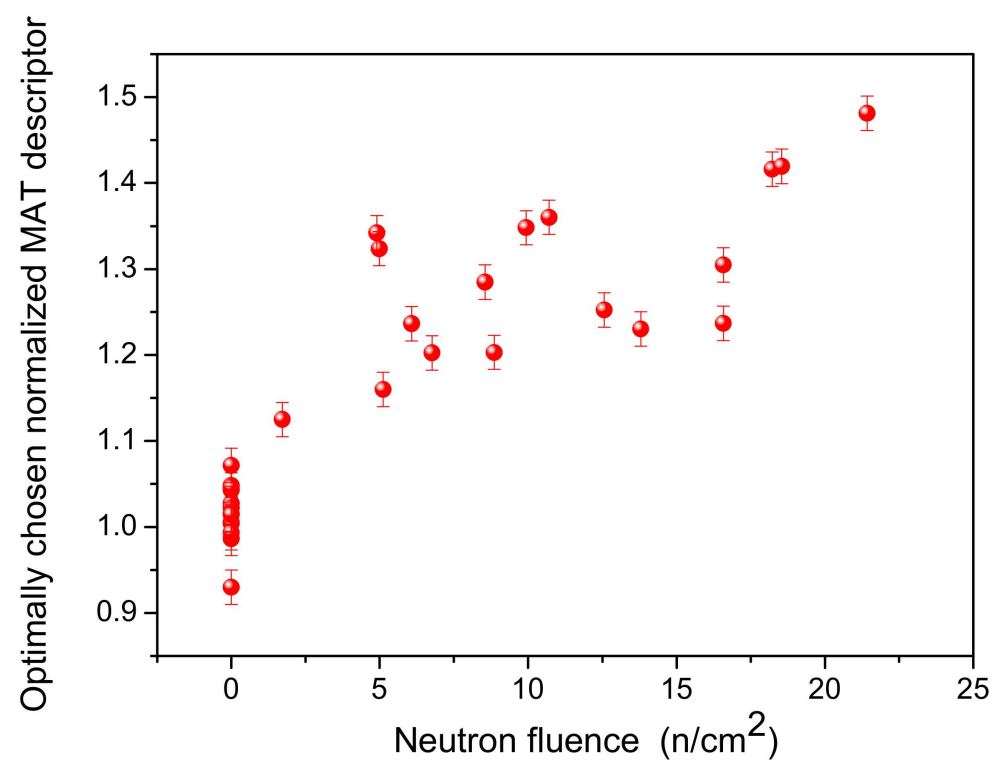

Figure 7. Optimally chosen MAT descriptor as a function of the neutron fluence, measured on base material (top and bottom sides).

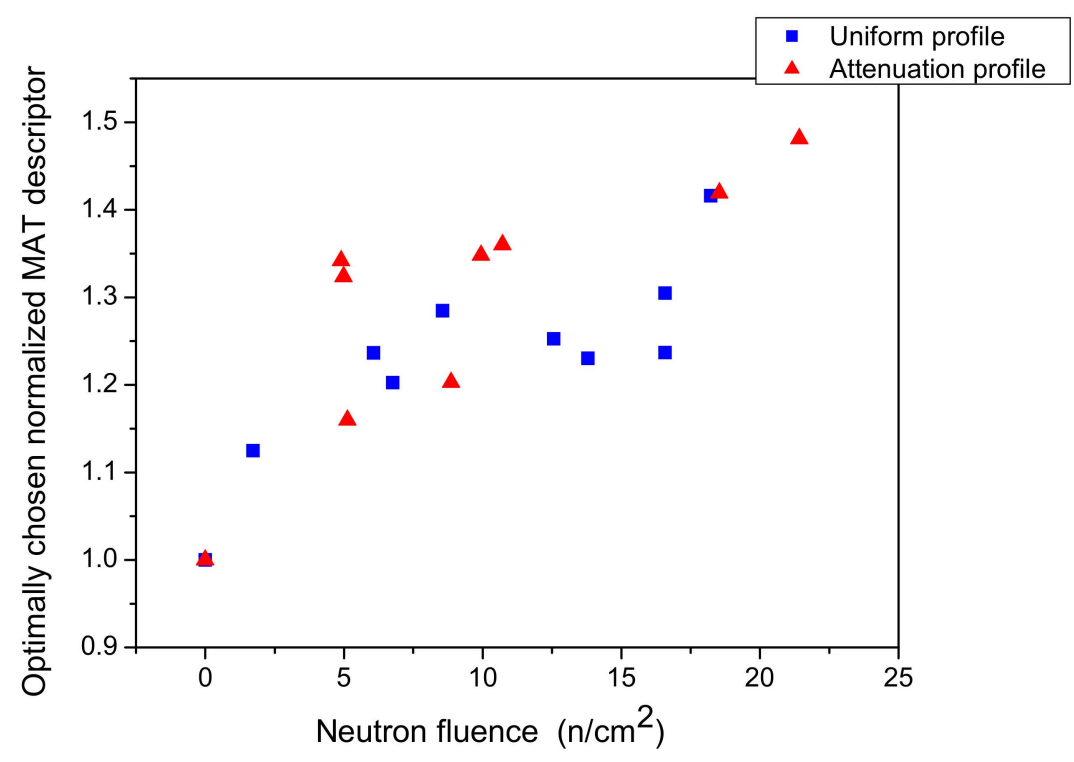

Figure 8. Optimally chosen MAT descriptor as a function of the neutron fluence, measured on base material, separating the samples irradiated by different neutron profiles. 


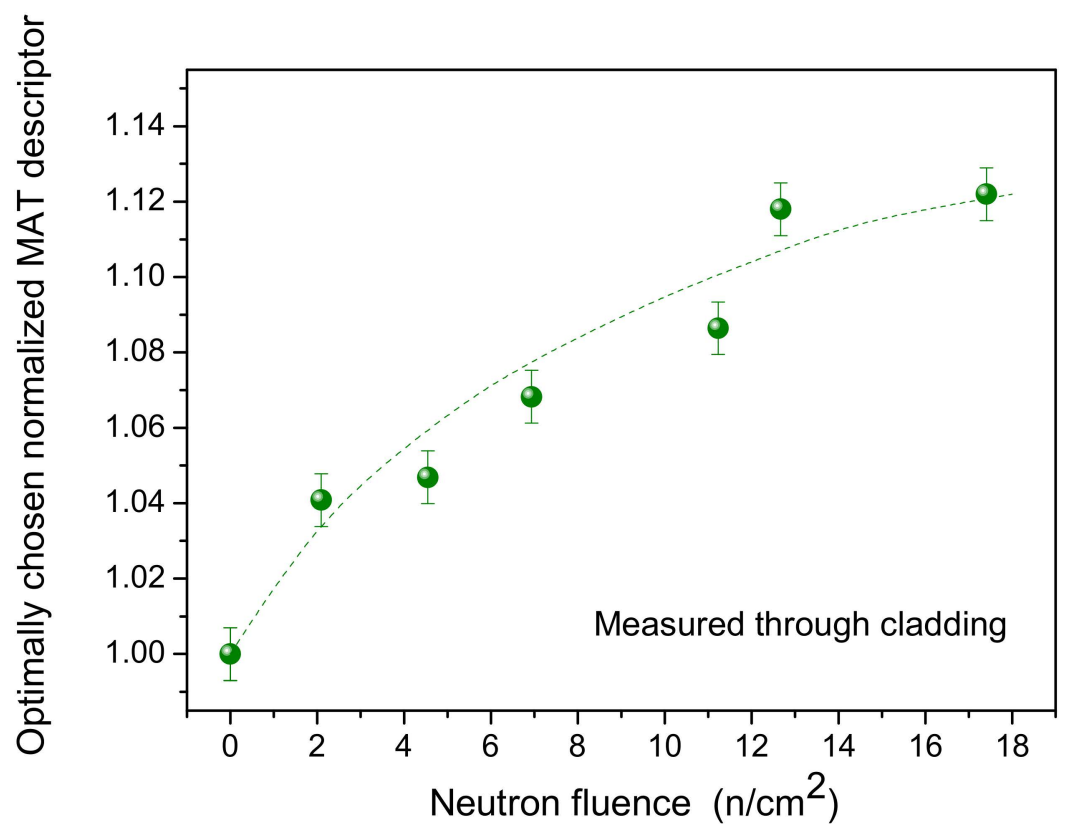

Figure 9. Optimally chosen MAT descriptor as a function of the neutron fluence, measured on the cladding. (The dashed line is added to the figure just to guide the eye.)

\section{Conclusions}

The influence of neutron irradiation on mechanical properties was investigated on reactor pressure vessel steel. Large blocks, cut from a real non-used reactor vessel, were irradiated at low irradiation temperature by neutrons in the BR2 reactor at SCK CEN. In this study, the blocks were measured by a nondestructive magnetic method, namely magnetic adaptive testing. The modification of the magnetic parameters as a function of the neutron fluence can be clearly seen. Although the effect of the irradiation on the cladding and on the base material cannot be separated, the weak magnetic properties of the cladding allows the base material to dominate in magnetic response. As an important result, the degradation of mechanical properties by the neutron irradiation can also be detected with a reasonable signal-to-noise ratio through the cladding, where the cladding acts as a nonmagnetic spacer, thus reducing measurement errors.

The experienced scatter of points on the base material could not be explained and needs further studies. However, this was not checked in detail and can only be detected by magnetic properties at the moment. It was shown that it is not a consequence of experimental error of the magnetic measurements. However, it is worth of mentioning that very similar scattering was found in previous studies on irradiated Charpy samples as well. We know that magnetic measurements could be sensitive to other unknown parameters, which have nothing to do with irradiation damage. The reason for the scatter detected by magnetic investigations needs further analysis.

An important conclusion of this work is that the magnetic technique is sensitive not only to irradiation damage but also to other unknown reasons that are masking the net irradiation effects.

Author Contributions: Conceptualization and original draft preparation: G.V.; investigation and methodology: G.V., A.G. and I.U.; project administration: A.G. and I.S. All authors have read and agreed to the published version of the manuscript.

Funding: This research was carried out under the framework of the "NOMAD" project. This project (Non-destructive Evaluation System for the Inspection of Operation-Induced Material Degradation in Nuclear Power Plants) received funding from the Euratom research and training programme 2014-2018 under grant agreement No. 755330.

Institutional Review Board Statement: Not applicable. 
Informed Consent Statement: Not applicable.

Data Availability Statement: The data are contained within the article.

Acknowledgments: Material was supplied by SCK CEN under the NOMAD project. The neutron irradiation of all the blocks were performed in the BR2 reactor at SCK CEN.

Conflicts of Interest: The authors declare no conflict of interest.

\section{References}

1. Koutsky, J.; Kocık, J. Radiation Damage of Structural Materials; Elsevier: Amsterdam, The Netherlands, 1994.

2. Ferreño, D.; Gorrochategui, I.; Gutiérrez-Solana, F. Degradation due to neutron embrittlement of nuclear vessel steels: A critical review about the current experimental and analytical techniques to characterise the material, with particular emphasis on alternative methodologies. In Nuclear Power-Control, Reliability and Human Factors; Tsvetkov, P., Ed.; IntechOpen Limited: London, UK, 2011; ISBN 9789533075990. Available online: http:/ / www.intechopen.com/articles/show/title/non-destructivetesting-for-ageing-management-of-nuclear-power-components (accessed on 14 May 2020).

3. Kronmüller, H.; Fähnle, M. Micromagnetism and the Microstructure of Ferromagnetic Solids; Cambridge University Press: Cambridge, UK, 2003.

4. Jiles, D.C. Magnetic methods in nondestructive testing. In Encyclopedia of Materials Science and Technology; Buschow, K.H.J., Ed.; Elsevier Press: Oxford, UK, 2001; p. 6021.

5. Tomáš, I. Non-destructive Magnetic Adaptive Testing of ferromagnetic materials. J. Magn. Magn. Mater. 2004, 268, 178-185. [CrossRef]

6. Tomáš, I.; Vértesy, G. Magnetic Adaptive Testing. In Nondestructive Testing Methods and New Applications; Omar, M., Ed.; IntechOpen: London, UK, 2021; ISBN 978-953-51-0108-6. Available online: http:/ / www.intechopen.com/articles/show / title/ magnetic-adaptive-testing (accessed on 10 July 2020).

7. Vértesy, G.; Gasparics, A.; Uytdenhouwen, I.; Szenthe, I.; Gillemot, F.; Chaouadi, R. Nondestructive investigation of neutron irradiation generated structural changes of reactor steel material by magnetic hysteresis method. Metals 2020, 10, 642. [CrossRef]

8. Tomáš, I.; Vértesy, G.; Gillemot, F.; Székely, R. Nondestructive Magnetic Adaptive Testing of Nuclear Reactor Pressure Vessel Steel Degradation. J. Nucl. Mater. 2013, 432, 371-377. [CrossRef]

9. Vértesy, G.; Gasparics, A.; Szenthe, I.; Rabung, M.; Kopp, M.; Griffin, J.M. Analysis of Magnetic Nondestructive Measurement Methods for Determination of the Degradation of Reactor Pressure Vessel Steel. Materials 2021, 14, 5256. [CrossRef] [PubMed]

10. Uytdenhouwen, I.; Chaouadi, R. Effect of neutron irradiation on the mechanical properties of an A508 Cl.2 forging irradiated in a BAMI capsule. Proc. ASME 2020, 1, PVP2020-21513, V001T01A060.

11. Uytdenhouwen, I.; Chaouadi, R.; NOMAD Consortium Members. Effect of neutron irradiation on the mechanical properties of an A508 Cl. 2 and 15kH2NMFA irradiated in the NOMAD_3 rig in the BR2 cooling water. Proc. ASME 2021, 1, PVP2021-61969, V001T01A024.

12. Uytdenhouwen, I.; Chaouadi, R.; NOMAD Consortium Members. NOMAD: Non-destructive evaluation (NDE) system for the inspection of operation-induced material degradation in nuclear power plants-Overview of the neutron irradiation campaigns. Proc. ASME 2020, 7, PVP2020-21512, V007T07A003. [CrossRef]

13. Vértesy, G.; Gasparics, A.; Szenthe, I.; Uytdenhouwen, I. Interpretation of Nondestructive Magnetic Measurements on Irradiated Reactor Steel Material. Appl. Sci. 2021, 11, 3650. [CrossRef]

14. Rabung, M.; Kopp, M.; Gasparics, A.; Vértesy, G.; Szenthe, I.; Uytdenhouwen, I.; Szielasko, K. Micromagnetic Characterization of Operation-Induced Damage in Charpy Specimens of RPV Steels. Appl. Sci. 2021, 11, 2917. [CrossRef]

15. Tomáš, I.; Kadlecová, J.; Vértesy, G. Measurement of flat samples with rough surfaces by Magnetic Adaptive Testing. IEEE Trans. Magn. 2012, 48, 1441-1444. [CrossRef] 